QUIPURAMAYOC $\mid$ Revista de la Facultad de Ciencias Contables

Vol. 20 N. ${ }^{\circ} 38$ pp. 189-205 (2012) UNMSM, Lima, Perú

ISSN: 1560-9103 (versión impresa) / ISSN: 1609-8196 (versión electrónica)

\title{
EL ASOCIATIVISMO COMO ESTRATEGIA PARA ENFRENTAR A UN MERCADO COMPETITIVO: UN ESTUDIO DE CASO DE LAS PEQUEÑAS EMPRESAS FARMACÉUTICAS
}

THE ASSOCIATIVISM AS A STRATEGY TO FACE UP A COMPETITIVE

MARKET: A CASE STUDY OF SMALL PHARMACEUTICAL COMPANIES

\section{María Luisa SaAvedra García * \\ Fernando Paúl Molina** \\ Deyanira Bernal Domínguez ${ }^{* * *}$}

Universidad Nacional Autónoma de México

[Recepción: Setiembre de 2012/ Conformidad: Octubre de 2012]

\section{RESUMEN}

El objetivo de esta investigación es demostrar la existencia de una necesidad real por parte de las empresas farmacéuticas, de asociarse con el fin de obtener ventajas comerciales y financieras derivadas de la compra conjunta y coordinación de las acciones de marketing. La metodología utilizada consistió es el análisis de caso tomando como unidad de análisis una "Asociación de Farmacias" de Argentina. Los hallazgos principales de esta investigación revelan que es posible aplicar el asociativismo en este tipo de organizaciones y que es posible lograr una acción coordinada de mercancías y la aplicación de estrategias de Marketing, siendo más las ventajas que las desventajas de aplicar estas herramientas.

Palabras claves: Asociativismo, estrategias de marketing, gestión de mercancías, pequeña empresa.

\begin{abstract}
The objective of this research is to demonstrate the existence of a real need on the part of pharmaceutical companies to partner with in order to obtain trade and financial advantages resulting from joint purchasing and coordination of marketing activities. The methodology used was the analysis of a case, taking as unit of analysis a "Pharmacy Association" of Argentina. The main findings of this research show that it is possible to apply the associativism to this type of organizations and it is possible to achieve a coordinated merchandise action and applying marketing strategies. There are more advantages than disadvantages of applying this tool.
\end{abstract}

Keywords: Associativism, marketing strategies, merchandise management, small business.

* Doctora en Administración por la UNAM. Sub Jefa de la División de Investigación de la Facultad de Contaduría y Administración de la Universidad Nacional Autónoma de México. email:maluisasavedra@yahoo.com

** Maestro en Administración estratégica de negocios por la Universidad de Misiones, Argentina. Socio del estudio contable Molina, en Misiones, Argentina.

*** Doctora en Ciencias Sociales por la Universidad Autónoma de Sinaloa. Líder del Cuerpo Académico estudios fiscales y administrativos y profesora investigadora de tiempo completo de la Universidad Autónoma de Sinaloa. 


\section{INTRODUCCIÓN}

El estudio de este tema es importante dado que el marcado individualismo que caracteriza al empresario, prácticamente sin distinción de actividad, ha llevado a un retraso muy significativo en la economía de la región teniendo en cuenta los recursos disponibles, tanto humanos como naturales. Es por ello que el impulso de este tipo de estrategias permitirá limar diferencias, aprender a obtener objetivos en conjunto y alcanzar un desarrollo como nación, si se tiene en cuenta un marco más amplio.

Los propósitos generales de la investigación son, por un lado demostrar la existencia de una necesidad real por parte de las empresas farmacéuticas de la Provincia de Misiones, Argentina de unirse para de esa forma obtener ventajas comerciales, y por el otro, cuantificar dichas ventajas derivadas de la compra conjunta y coordinación de las acciones de marketing.

\section{a. Planteamiento del problema}

El Asociativismo puede definirse como el acto de asociarse o la acción de unir fuerzas para un fin común. En una definición más amplia, es cualquier iniciativa formal o informal, que reúne a un grupo de empresas (o personas) con el objetivo de superar dificultades generando beneficios económicos, sociales o políticos. El hecho de asociarse permite a las empresas obtener economías de escala a través de la colaboración interempresarial, sin tener que reunir la inversión en una sola firma (Surraco, 2007).

La naturaleza de la sociedad asociativista, basada en la igualdad de los individuos y en la ayuda mutua frente a las necesidades comunes, contiene un conjunto de valores que permiten alcanzar niveles considerables de productividad, al potenciar los recursos humanos, económicos y técnicos a través del a sinergia de grupo (Elgue, 2005).

Una empresa con perfil asociativista comparte información e ideas, posee gestión transparente, busca resultados de largo plazo, toma decisiones colegiadas, ve en el competidor o proveedor a un aliado con quien poder aprender a crecer.

Por lo anterior nos permitimos formular las siguientes preguntas de investigación:

¿Es posible aplicar un control de inventarios con reorden y demanda incierta $o$ bien de demanda constante, mantenido en forma conjunta por un grupo de empresas farmacéuticas en la Región del Alto Paraná Misionero sin perder su individualidad jurídica? Por otra parte, ¿Es posible el desarrollo y aplicación de estrategias de marketing en forma conjunta por parte de estas empresas?

La importancia de este problema queda expresada en los beneficios que se obtendrían a partir de su resolución. Beneficios tanto para los consumidores, quienes tendrían acceso a medicamentos a menores precios como así también a un mejor servicio brindado por personal capacitado; consecuentemente generaría beneficios para las empresas asociadas ya que lograrían diferenciarse de sus competidores ampliando de esta forma su participación en el mercado y consecuentemente mejorando sus resultados.

\section{b. Objetivo general}

Determinar si es posible la aplicación de un sistema de control de inventarios con reorden y demanda incierta o bien de demanda constante, mantenido en forma conjunta por un grupo de empresas farmacéuticas en la Región del Alto Paraná Misionero sin perder su individualidad jurídica. Así como proponer la aplicación de estrategias de Marketing en forma conjunta. 


\section{c. Metodología}

\section{c.1 Diseño de la investigación y funda- mentación}

El diseño de la investigación es no experimental y transversal, dado que no se manipularán las variables y los datos se toman de la realidad tal cual se presentan y en un solo momento del tiempo. Aplicándose también, un estudio de caso simple, teniendo como unidad de estudio a un grupo de empresas farmacéuticas de la provincia de Misiones, Argentina y como unidad de análisis al asociativismo.

\section{c. 2 Procedimientos y técnicas de recolec- ción de datos}

Los procedimientos utilizados para recoger datos primarios fueron los siguientes:

El de observación simple, el cual se caracteriza por evitar inhibiciones o alteraciones en la conducta de los sujetos observados, ya que se tratará de pasar lo más desapercibido posible.

La entrevista informal. Consistente en situarse frente al investigado y formularle preguntas, a partir de cuyas respuestas habrán de surgir los datos de interés.

Las fuentes utilizadas para los datos secundarios fueron: archivos, documentos, páginas web, entre las principales.

\section{ANTECEDENTES DE LA INVESTIGACIÓN}

En la actualidad existen figuras de asociativismo entre pequeñas empresas en diferentes países del mundo con es el caso de Japón, que lleva muchos años fomentando esta figura con mucho éxito (Ibata-Arens \& Obayashi, 2006). En este estudio tomaremos como modelo el caso de Brasil, por ser un páis la- tinoamericano con un contexto similar al de Argentina.

\subsection{Antecedentes en el Brasil}

Se toma el caso de Brasil, con el fin de determinar que estrategias de éxito se pueden aplicar al caso de Argentina. Febrafar (Federación Brasileña de Redes Asociativas de Farmacias) fue fundada en Febrero del 2000 y constituida como sociedad civil sin fines de lucro con sede administrativa en la ciudad de San Paulo. La misma esta regida por estatuto social, y tiene actuación en todo el territorio nacional (once estados más el Distrito Federal).

Actualmente, Febrafar congrega 34 redes de farmacias independientes $(5,300$ puestos de ventas en 19 estados y cerca de 1,500 municipios), en donde cada farmacia factura en promedio 45 mil reales mensuales. En el 2004, la facturación de los negocios integrados a las redes asociativas de Febrafar alcanzó los 1,3 billones de reales (Tamascia, 2005).

Febrafar tiene como misión promover la integración y el fortalecimiento de las redes asociadas en todo el territorio nacional, buscando el desarrollo socioeconómico y la representatividad política de sus afiliados.

A Febrafar se le atribuyen los siguientes compromisos.

- Sistematizar la información e unificar las redes asociadas.

- Orientar a los asociados sobre la realidad económica, tecnológica y política del sector, mediante el planeamiento estratégico para acompañar los cambios del mercado.

- Acompañar y contribuir a la expansión y divulgación de los servicios de cada red y, sobretodo, al éxito del proceso productivo como un todo.

- Beneficiar a los empleados y colaboradores de las redes asociativas, ofreciendo ac- 
tualización y capacitación profesional.

- Promover eventos (congresos, simposios, workshops, encuentros y ferias de negocios) para estrechar relaciones en la cadena farmacéutica (industria, distribuidor y minorista).

Para garantizar una buena prestación de los servicios y la atención a sus asociados, Febrafar dispone de un Directorio Ejecutivo y de dos Consejos (Ética y Fiscal).

Moreira (2005), revela que en Brasil posee 51,218 farmacias comerciales. Las pequeñas farmacias representan el 86,6\% (44,386 establecimientos) y las medias y grandes farmacias $13,4 \%$ (6,832 establecimientos). Señala también que la expansión de las redes de farmacias y droguerías ocurrió en la década de los 80, a partir de la informatización de los stocks de los puntos de venta y de la disminución en el número medio de empleados por negocio.

Las farmacias independientes representan más del $85 \%$ del total de las farmacias brasileñas. Ellas están, cada vez más, actuando en base al modelo asociativo de gestión empresarial ya que el $6 \%$ de las farmacias independientes se encuentran asociadas. Atienden bajo una misma bandera y se organizan en redes para enfrentar las dificultades del mercado dominado por las grandes corporaciones.

Entre las alternativas adoptadas por el minorista para competir con las grandes redes del sector se destacan:

- Fomento de una política de mejora en los precios.

- Mejora en la calidad de la atención.

- Promoción de servicios adicionales.

- Diversificación del mix de productos.

- Desenvolvimiento de productos con marcas propias.
- Incentivo de la actividad asociativa.

Hoy una buena asociación no busca solo lograr descuentos comerciales de los distribuidores. Hay que considerar, también, proyectos sobre genéricos, capacitación y cursos, centro de compras y de convenio, integración de datos, inversión en marketing, etc. Son los componentes adicionales los que distinguen a una asociación de otra, y es por eso que las redes profesionalizadas poseen asociados exitosos. Por lo que corresponde decir que: "No basta con ser asociativista para ser bueno, sino que es fundamental ser bueno para ser asociativista" (Tamascia, 2005).

A modo de ejemplo concreto de una red brasilera es posible mencionar a Agafarma fundada el 3 de abril del 1997 en el Estado de Grande do Sud, la cual nació de la necesidad de lograr una unión y un fortalecimiento por parte de un grupo de empresarios, para hacer frente a los crecientes desafíos del mercado surgidos a partir de la globalización de la economía (Scott, 2006).

Esta alianza estratégica de pequeños y medios empresarios, a través del asociativismo, fue la alternativa elegida para crecer dentro de una acérrima competencia.

Hoy, además de constituir un modelo de creatividad y agresividad en el mercado, Agafarma es el símbolo de una realidad que tuvo éxito en el mercado minorista farmacéutico "gaucho".

La administración de la red esta conformado por:

- Un Gestor de Negocios, que puede ser director o asociado de la red.

- Dos empleados negociadores.

- El apoyo del equipo de Marketing.

- El apoyo del consejo de la asociación.

Funcionamiento 
- Las farmacias que no están registradas o bajo convenio no pueden vender en la red.

- Las empresas proveedoras interesadas en vender a la red deben estar habilitadas.

- Deben formalizar su participación en la sociedad.

- Deben entregar una comisión variable conjuntamente con un informe.

\section{Negociación Principal}

- Para periodos de 6 a 8 meses se solicitan propuestas a los principales distribuidores, informándose previamente los ítems de mayor importancia.

- Las propuestas son analizadas por una comisión de asociados.

- Posteriormente se seleccionan los proveedores.

- Seguidamente se comunica a los restantes asociados la decisión tomada.

- Se procede al cobro de una cuota a los proveedores.

\section{Relación Comercial}

- Se estipula un porcentaje a comprar a cada proveedor.

- Se efectúa un seguimiento de las compras de cada uno de los asociados.

- Se felicita a los asociados que cumplen.

- Se solicita explicaciones a quien no cumple.

- Se toman medidas en los casos de incumplimiento: advertencia, multa, exclusión, etc.

\section{Penalidad a los Proveedores}

- Raramente ocurren problemas con los proveedores.

- En casos raros de incumplimiento de las comisiones que son negociadas, se pro- duce una cancelación del contrato, que es comunicado a la Red para que no compre más productos a ese proveedor.

\section{Control}

- Se utilizan los informes de las propias distribuidoras que por muestreo son chequeados, eventualmente, junto a los asociados.

- Se informa a los asociados cuanto es su cuota de compra.

Agafarma ofrece a sus clientes promociones, tarjetas de crédito propias y productos específicos para cada necesidad, con calidad y a precios competitivos y, lo que es más importante, honestidad en todo lo que hace.

La Red Agafarma comercializa bajo marca propia, la línea infantil Agababy, productos de higiene, fabricados por prestigiosas industrias del país, siguiendo los más altos criterios de calidad.

\subsection{Antecedentes en la Argentina}

La Unión Argentina de Redes de Farmacias es la primera alianza, de carácter nacional, integrada por los principales grupos Farmacéuticos de mayor trayectoria y relevancia comercial de la República Argentina (Taleb, 2006).

Nuclea en todo el país a las Redes de Farmacias con mayor nivel de facturación, variedad de servicios, calidad en la prestación farmacéutica y soporte al Sistema de Salud, concentrado en cada plaza la atención de las principales Obras Sociales y Prepagas del país.

Es una Asociación Civil, sin Fines de Lucro, integrada por "Redes Socias" con igual nivel de representatividad y con total compatibilidad de intereses.

Proporciona a la "Red Socia" exclusividad en cada plaza ya que brinda: 
o Adecuada Cobertura Nacional, con Cantidad Óptima de Locales.

o Exige unanimidad de aceptación entre sus socios para la incorporación de una nueva Red.

o Localmente las Redes conservan su propia marca comercial, ya que así son reconocidas por sus clientes, profesionales, proveedores e instituciones, recibiendo la confianza de todos ellos por la calidad y seguridad en la prestación de servicios.

o Las Redes integrantes de UARFA, exhiben una identificación común como símbolo de pertenencia y aval de garantía de la Institución

UARFA propone trabajar en conjunto con la industria y la distribución farmacéutica con una contraprestación real de servicios, que permita a través de cada acción, alcanzar un mayor volumen de ventas global. No es un centro de compras, ni tampoco un "Broker de Farmacias" que sólo pretende negociar puntos de rentabilidad.

Constituye la primera Mega Red empresario farmacéutica que responde con una organización nacional a la evolución y maduración del Retail Farmacéutico.

Potencia la fortaleza local de cada red impulsando el desarrollo de negocios a escala nacional. Brinda además, institucionalmente la representatividad conjunta frente a los distintos sectores del mercado de salud, facilitando y centralizando la gestión comercial y su comunicación.

Garantiza la efectividad de la implementación de las acciones comerciales en todo el país, ofreciendo cooperación y costos-eficientes en el mercado de Redes farmacéuticas.

Las Redes de Farmacias asociadas, tienen como característica la de adoptar figuras jurídicas clásicas tales como la de Sociedad
Anónima, Sociedad de Responsabilidad Limitada, etc. Sin embargo, ninguna bajo la forma de Agrupación de Colaboración de Empresas o Sociedades Cooperativas.

Es más, este tipo de figuras tienen vedado el ingreso a la Unión, y la agrupación de Redes socias bajo estas formas es motivo de expulsión.

Un ejemplo concreto de agrupación de empresas farmacéuticas en la Argentina sería Phârmakus. Se trata de un grupo empresario que nuclea a sesenta y cinco de las farmacias tradicionales más importantes de la Capital Federal y el Gran Buenos Aires, la mayoría con más de cuarenta años de actuación profesional (Albornoz, 2005).

Se trata de una unión comercial que reúne entidades de reconocida trayectoria, que recupera lo mejor en cuanto a calidez y calidad en el servicio que sabe dar el farmacéutico de siempre.

La globalización y el momento que atraviesa la Argentina imponen cambios en las sociedades y en la atención de sus necesidades y demandas, cambios que los miembros del grupo han decidido acompañar mediante la creación de una red para poder garantizarle a cada cliente siempre la mejor y más completa atención profesional.

Cada una de las farmacias agrupadas tiene un nombre, una marca reconocida y exitosa, que el cliente conoce y lo acompaña en el cuidado de su salud desde hace décadas. Phârmakus quiere ser un apellido para agregar al buen nombre de cada una de las farmacias que la integran, y apunta a poner en primer lugar la atención al cliente para que éste sienta confianza y respaldo en la adquisición de sus medicamentos.

Cada una de las sesenta y cinco farmacias que integran el grupo, realizan aproximadamente un ochenta porciento de sus compras en una droguería de nombre Siete Farmas. 
Siete Farmas es una sociedad anónima que posee como accionistas a varios empresarios farmacéuticos de la provincia de Buenos Aires, y dispone de alrededor de 8.000 artículos.

Mediante la celebración de un convenio comercial y el pago de una cuota mensual de 200 pesos más IVA, cada una de las farmacias queda luego habilitada a realizar sus compras a la droguería mencionada más arriba. Esta luego, al realizar la venta, procede a retener un porcentaje que oscila de entre el 1 al 1.5 por ciento del monto total en concepto de impuestos. Mediante esta modalidad las farmacias logran incrementar su margen de utilidad en un diez por ciento.

En la Provincia de Misiones podemos encontrar un grupo de empresarios farmacéuticos pertenecientes a las localidades de Eldorado, Puerto Esperanza, Wanda, Puerto Iguazú y San Vicente, que poseen como característica común el hecho de ser lideres en los mercados locales en los que operan. Esto ha posibilitado la unión entre ellos, ya que al no ser competidores directos, están más predispuestos a trabajar en conjunto fundamentalmente en lo que ha compras a proveedores se refiere.

Es decir se trata de una etapa muy temprana del proceso de creación de una Agrupación de Colaboración de Empresas en el Rubro de Farmacias, más aún si nos basamos en la experiencia de las Redes de Farmacias en el Brasil. Es decir, prácticamente todas estas se han iniciado de una manera informal para luego evolucionar en estructuras más complejas, en cuanto a funcionamiento $y$ operaciones.

Los empresarios misioneros, al ser líderes en sus respectivas localidades, no han tenido una experiencia muy feliz a la hora de invitar a participar de sus operaciones de compra, a otros empresarios más peque- ños. Esto se ha debido a fundamentalmente a que estos empresarios más pequeños al tener problemas financieros, lo cual constituye uno de los principales requisitos a cumplir por parte de los miembros de una Red, no pueden disponer de un buen inventario de productos para satisfacer la demanda de la mayoría de los clientes por un lado y por otro no les es posible por ejemplo mantener sus locales abierto las 24 horas al público por no poder afrontar los mayores costos que implicaría la contratación de profesionales adicionales y el pago de las correspondientes horas extras. Ante esta realidad los empresarios más pequeños optan por denunciar a los empresarios más grandes ante el Colegio de Farmacéuticos de la Provincia de Misiones, esgrimiendo por ejemplo la falta de la presencia de un farmacéutico durante las horas extras en las que permanecen abiertos al público las farmacias, muchas veces sin que ello sea cierto. Entonces la posibilidad de que se agrupen empresarios grandes y pequeños en un clima de desconfianza, como el que se genera al existir denuncias cruzadas, es muy lejana.

\section{MARCO TEÓRICO}

\subsection{Marco Jurídico del Asociativismo}

Las figuras jurídicas alternativas a adoptar por el conjunto de empresarios decididos a comenzar a trabajar en forma asociativa sin que ello implique perder su individualidad jurídica, se analizaron de autores tales como Perciavalle, M. (2000), Nissen, R. (1985), Marsicano, A. y Marsicano, S. (2004), Prensa Cooperativa (2006), San Pedro, J. (1987), Talavera, O. (2005), Veron, A. (1998), Zaldivar, Manovil y Ragazzi (1997), Barreira, D. (2000), Favier, 1988), Karschenboim, R. (2001), Le Pera, S. (1984), Otaegui, j. (1984). 
María Luisa SaAVEdra García

Fernando Paúl Molina/ Deyanira Bernal Domínguez

Cuadro 1. Principales tipos de relaciones de cooperación empresarial

\begin{tabular}{|c|c|c|c|}
\hline Criterio & Tipos & Descripcion & Autores \\
\hline \multirow[t]{4}{*}{$\begin{array}{l}\text { Forma en que se } \\
\text { vinculanlos } \\
\text { participantes }\end{array}$} & Honzontal & $\begin{array}{l}\text { Las empresas están en la misma etapa } \\
\text { de la cadena de valor ylo etapas de } \\
\text { producción. }\end{array}$ & $\begin{array}{l}\text { Menguzzato (1992), Femandes } \\
\text { da Silva (2005) }\end{array}$ \\
\hline & Vertical & $\begin{array}{l}\text { Interacción entre empresas de las } \\
\text { diversas etapas de producción }\end{array}$ & $\begin{array}{l}\text { Menguzzato (1992), Femandes } \\
\text { da Silva (2005) }\end{array}$ \\
\hline & Simbiotica & $\begin{array}{l}\text { Las empresas de diferentes areas } \\
\text { estrategicas e industrias se } \\
\text { complementan para crear y aprovechar } \\
\text { sinergias. }\end{array}$ & $\begin{array}{l}\text { Menguzzalo } \\
\text { Casanueva (2003) }\end{array}$ \\
\hline & Comensalista & $\begin{array}{l}\text { Empresas del sector realizan las } \\
\text { mismas demandas de recursos a su } \\
\text { entomo }\end{array}$ & Casanueva (2003) \\
\hline \multirow[t]{2}{*}{$\begin{array}{l}\text { Naturaleza del } \\
\text { marco en el cual } \\
\text { seconstituyen }\end{array}$} & Formales & $\begin{array}{l}\text { La cooperación se manifiesta en } \\
\text { obligaciones contractuales y estructuras } \\
\text { formales de control y autoridad. }\end{array}$ & Hagedon (1993) \\
\hline & Informales & $\begin{array}{l}\text { Producto de la convicción en la buena } \\
\text { voluntad de los participantes y creencia } \\
\text { que estos se esforzarán por lograr los } \\
\text { objetivos comunes. }\end{array}$ & Sebastiàn (2000) \\
\hline \multirow{2}{*}{$\begin{array}{l}\text { Naturaleza de los } \\
\text { recursos que se } \\
\text { comparten }\end{array}$} & Estructurada & $\begin{array}{l}\text { Involucran inversión o participación de } \\
\text { capital }\end{array}$ & Menguzzato (1992) \\
\hline & No estructurada & Ausencia de participación de capital & Menguzzato (1992) \\
\hline \multirow[t]{5}{*}{$\begin{array}{l}\text { Propósito de la } \\
\text { relación }\end{array}$} & $\begin{array}{l}\text { Objetivos de } \\
\text { mercado }\end{array}$ & $\begin{array}{l}\text { Desartollar un nuevo mercado. } \\
\text { posicionarse en un mercado }\end{array}$ & $\begin{array}{l}\text { Johnsen y Johnsen (1999), } \\
\text { Sebastian }(2000)\end{array}$ \\
\hline & $\begin{array}{l}\text { Transterencia } \\
\text { tecnoldgica }\end{array}$ & Acceso a nueva tecnologia o diferente & Sebastiàn (2000), Gallie (2005) \\
\hline & $\begin{array}{l}\text { Investigacion } \quad y \\
\text { desarrollo }\end{array}$ & $\begin{array}{l}\text { Compartir los costos en investigación y } \\
\text { desarrollo }\end{array}$ & Sebastian (2000), Gallié (2005) \\
\hline & internacionalizaoon & Desarrolio de un mercado extranjero & 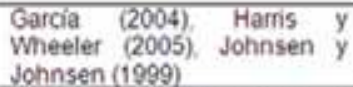 \\
\hline & $\begin{array}{l}\text { Crecimiento de la } \\
\text { empresa }\end{array}$ & $\begin{array}{l}\text { Búsqueda de mejores operaciones, } \\
\text { mayores ventas y condición de negocio. }\end{array}$ & $\begin{array}{l}\text { Sebastiân (2000). Singh y } \\
\text { Mitchell (2005) }\end{array}$ \\
\hline \multirow[t]{2}{*}{$\begin{array}{l}\text { Ambito } \\
\text { geografico }\end{array}$} & Local o nacional & $\begin{array}{l}\text { Se establecen con empresas de la } \\
\text { misma localidad pais o región }\end{array}$ & Sebastiàn (2000) \\
\hline & Intemacional & $\begin{array}{l}\text { Se establecen con empresas de otro } \\
\text { pais }\end{array}$ & Sebastian (2000) \\
\hline \multirow[t]{2}{*}{ Duración } & Permanente & Relación de largo plazo & \multirow[t]{2}{*}{ Sebastiàn (2000) } \\
\hline & Temporal & $\begin{array}{l}\text { Relación de corto plazo. Generalmente } \\
\text { acotada por la duración de un proyecto } \\
\text { especifico o logro de un objetivo }\end{array}$ & \\
\hline \multirow{4}{*}{$\begin{array}{l}\text { Especificidad de } \\
\text { los recursos de la } \\
\text { red y su } \\
\text { continuidad }\end{array}$} & Agregacion & Se comparten recursos & \multirow[t]{4}{*}{ Doulard y Heitz (2003) } \\
\hline & Heunisticas & $\begin{array}{l}\text { Producción conjunta de un valor } \\
\text { especifico }\end{array}$ & \\
\hline & Transaccionales & Existe complementanedad & \\
\hline & Orquestación & Favorecen la especialización & \\
\hline \multirow{3}{*}{$\begin{array}{l}\text { En funcion del } \\
\text { aprendizaje que } \\
\text { se espera } \\
\text { obtener }\end{array}$} & Comerciales & $\begin{array}{l}\text { Explotación de conocimiento y } \\
\text { capacidad existentes }\end{array}$ & \multirow[t]{3}{*}{ Bnones, Ramirez y Ros (2006) } \\
\hline & Hibridas & $\begin{array}{l}\text { Persiguen simultaneamente } \\
\text { oportunidades para capturar valor de las } \\
\text { capacidades y activos existentes y } \\
\text { actividades de crear nuevo valor }\end{array}$ & \\
\hline & De aprendizaje & $\begin{array}{l}\text { Fuertes deseos de exploración y } \\
\text { búsqueda de nueva información }\end{array}$ & \\
\hline
\end{tabular}

196/ QUIPUKAMAYOC | Vol. 20(38) 2012 
2.1.1 Asociativismo como alternativa para prosperar en un mercado competitivo

El secreto es no prever el futuro, sino prosperar en un futuro que no puede ser previsto. Tenemos que estar preparados para cualquier cosa que pueda suceder.

La cooperación empresarial lo que busca es la creación de valor a través del trabajo común de dos o más empresas jurídicamente distintas que interactúan con la finalidad de generar sinergias. Así, Douard y Heitz, 2003 (citados en Ojeda, 2009), señalan que no se trata de un simple intercambio y en sus distintas manifestaciones puede abarcar solo una parte de la totalidad de actividades que realiza una empresa. En el siguiente cuadro se muestra los principales tipos de relación que puede establecerse entre empresas pequeñas.

\subsubsection{Puntos Fuertes del Asociativismo}

$\mathrm{El}$ asociativismo como movimiento presenta los siguientes puntos fuertes:

- Unión: La unión de personas en torno de un mismo objetivo permite el fortalecimiento del grupo en la búsqueda de soluciones comunes. El cambio de paradigmas que el modelo trae hace que la visión de competencia cambie, permitiendo que aquél que era visto como competidor pase a ser un aliado. Ese comportamiento de compañerismo fomenta el intercambio de experiencias y conocimiento entre los asociados, permitiendo así el crecimiento de todos.

- Cultura emprendedora: En el modelo asociativista es común la realización de reuniones, asambleas, encuentros de negocios y otras actividades en grupo. Esta práctica fomenta el espíritu emprendedor de los asociados, haciendo que pasen a discutir sobre la situación y perspectivas del mercado farmacéutico y no solo sobre las cuestiones propias de sus establecimientos. Es decir pasen a tener una visión del conjunto.

- Capacitación. Los empresarios y sus empleados optan por capacitarse, con el fin de hacer frente a la competencia de las grandes empresas. Esto permite obtener mejoras significativas en la gestión del negocio, en la calidad del trabajo y de la atención al cliente.

- Fijación de una marca. La utilización de una marca única por parte de un grupo de empresas es uno de los éxitos del modelo y permite la reducción de los costos de divulgación de la misma. La existencia de una marca única permite la celebración de convenios o contratos, trabajar con una tarjeta de crédito en la que figure el nombre de la red, como así también la confección de material para el apoyo promocional del establecimiento. Así también, la marca única permite a su vez que el grupo comercialice productos de marca propia.

- Compra conjunta: El modelo asociativista no permite la compra centralizada, pero al representar la asociación volúmenes significativos es posible obtener descuentos y demás beneficios para el grupo. Los acuerdos o negociaciones entre los asociados y los proveedores ocurren en todos los niveles, es decir, a nivel de distribuidores y fabricantes.

- Otros acuerdos o negociaciones: Por formar parte de una red es posible establecer acuerdos o negociaciones con:

- Instituciones Bancarias.

- Empresas de software 
- Gráficas

- Agencias de Propaganda y Publicidad

- Proveedores de muebles y equipamientos para los establecimientos comerciales

El asociativismo es formado por un grupo de empresas, pero la asociación es formada por un grupo de de personas.

\subsection{3 ¿Qué es una cooperativa?}

La Alianza Cooperativa Internacional (ACI), máximo organismo mundial que nuclea a aproximadamente 800 millones de cooperadores, con la intención de establecer los lineamientos mínimos que son comunes a toda cooperativa, ha definido doctrinariamente aesta entidad solidaria de la siguiente manera: "Una cooperativa es una asociación autónoma de personas agrupadas voluntariamente para satisfacer sus necesidades económicas, sociales y culturales comunes, por medio de una empresa que se posee en conjunto y se controla democráticamente" (Prensa Cooperativa, 2006).

Con respecto a las implicaciones tributarias se analizó la gravabilidad de la figura jurídica elegida ante los impuestos nacionales y provinciales según la alternativa de gestión de inventario a adoptar, es decir la compra directa y a nombre propio de los medicamentos y mercaderías por parte de la agrupación para su posterior venta a los asociados o bien considerando únicamente la función de mero negociador entre los laboratorios, droguerías, distribuidores y los miembros asociados.

\subsection{La gestión de inventarios}

Se analizaron las ventajas y desventajas de cada una de las alternativas de gestión de inventarios como así también sus implicancias tributarias según la figura jurídica adoptada. Es por ello que, y con relación al primer tema mencionado, se analizó el modelo de lote económico de compra y el sistema Just in Time (Aquilano, N., Chase, R., Jacobs, R., 2004, Eppen, G., Gould F., Shmidt, C. Moore y Jeffrey H. y Watherford, L., 2000).

\subsubsection{Lote económico de compra}

Es importante tener en claro que la gestión de inventarios tiene asociado varios costos. En general, hay tres tipos de costos asociados a la actividad de inventarios: Costos de Existencias, Costos de Pedidos y Costos de Faltantes. Por el hecho de mantener inventario, se está renunciando a la oportunidad de hacer otras inversiones y obtener una renta asociada. Esto, que se conoce como el Costo de Oportunidad, es quizá el componente más importante del costo de mantenimiento de inventarios (Epen, et al., 2000). La magnitud de dicho costo está estrechamente relacionada con la tasa de interés o renta que generan las inversiones alternativas.

En cuanto a los Costos de los pedidos, cada vez que se hace un pedido para reabastecer los inventarios, se incurre en un costo de pedido. Este costo es independiente de la cantidad de unidades incluidas en el pedido. Está relacionado con la cantidad de tiempo empleado en preparar la documentación, llevar la contabilidad correspondiente y en presentar el pedido. Por lo anterior es conveniente aplicar el método del lote económico de compra con el fin de encontrar la cantidad óptima a comprar en cada pedido, que permita minimizar tanto los costos de mantenimiento como los costos pedir, considerando también un nivel mínimo de inventar que cubra a la empresa del riesgo de quedar desabastecido ${ }^{1}$.

4 Para ver una explicación más amplia de la aplicación de este método ver: Saavedra, 2011. 


\subsubsection{Just in time}

El Just in Time es una filosofía empresarial que se concentra en eliminar el despilfarro en todas las actividades internas de la organización y en todas las actividades de intercambio externas. Esta definición establece la idea clave del Just in time -eliminación del despilfarro-, lo cual exige eliminar todos los insumos de recursos que no añaden valor al producto o servicio. El objetivo es proporcionar satisfacción al cliente al tiempo que se minimiza el costo total. Ésta es la esencia del proceso Just in time, que integra el compromiso total de calidad.

La educación JIT / CTC (Just in time / control total de calidad) desarrolla las habilidades de estas personas y fomenta su creatividad. Además de capacitar y educar a los empleados, la alta gerencia debe reformar la cultura de la empresa. La meta es derribar los obstáculos que estorban la comunicación y les impiden a los trabajadores cooperar y confiar entre sí y confiar en sus clientes y proveedores.

\subsection{Estrategias de Marketing}

Se consideraron las estrategias de marketing a seguir en términos de Marca, Precios y Canales de Distribución consultando autores tales como Kotler, Armstrong (1996) y Braidot, N. (2005).

\subsubsection{Estrategias de Marca}

En mercados cada vez más globalizados y competitivos, la marca es el principal factor de diferenciación. Una marca representa la promesa del vendedor de entregar, de manera consistente, una serie específica de características, beneficios y servicios a los compradores.

Las mejores marcas transmiten una garantía de calidad. Una marca puede ofrecer hasta cuatro grados de significado (Kotler y Armstrong,1996):

- Los atributos: Una marca primero despierta en la mente ciertos atributos del producto.

- Los beneficios: Los clientes no compran atributos, compran beneficios. Por consiguiente los atributos se deben traducir a beneficios funcionales y emocionales.

- Los valores: Una marca también dice algo respecto de los valores de los compradores.

- La personalidad: Una marca también proyecta personalidad. Si esta marca fuera una persona ¿Qué tipo de persona sería?

Todas las empresas quieren construir marcas fuertes. Es imposible calcular el valor de una marca hasta que aparezcan varios compradores pujando por adquirir el nombre de la marca. Y los compradores interesados diferirán en lo que ofrecerán para comprar una marca. Cada empresa tiene que decidir las medidas del mercado que indican aumentos o disminuciones en el valor de marca. El hecho es que una marca evoca una serie de expectativas de los consumidores. El valor de marca resulta de lo bien, o mal, que se satisfacen las expectativas de los consumidores. Cuanto mayor es la satisfacción, mayor será el valor de marca. Y cuanto mayor es el valor percibido de la oferta, mayor es el valor de marca.

Las Cadenas de Farmacias, no solo en la Argentina sino también en Latinoamérica, se basan en el Marketing, es decir se trata de un formato basado en el aspecto comercial. La Farmacia Mediana se ubica en el mercado con un modelo mixto, un modelo de transición entre el Marketing y el Servicio. Lo que es posible observar habitualmente, es que la Farmacia Mediana comienza a animarse a entrar, a imitar o a iniciar acciones comer- 
ciales similares a las de las Cadenas de Farmacias. La Farmacia Pequeña se ha quedado sustentada en el Servicio, es decir en un formato básicamente de prestación de servicio.

\section{Estado Actual de las empresas del Sec- tor en Argentina (CILFA, 2006)}

\subsection{Industria Farmacéutica}

Es un sector industrial y empresarial dedicado a la investigación, desarrollo, producción y comercialización de medicamentos para el tratamiento y prevención de las enfermedades, y el cuidado de la salud en general (Romero, 2005).

\subsection{Estructura Productiva y Cadena de Valor}

En términos generales, la cadena de valor de la industria farmacéutica se compone por tres eslabones: a) los laboratorios, que llevan a cabo actividades de I\&D y producción de moléculas básicas, principios activos y medicamentos, b) la distribución, cuyos principales agentes son las distribuidoras y droguerías, y c) la venta o reparto final, a cargo de hospitales, clínicas, sanatorios, farmacias, supermercados, kioscos y otros actores de menor peso. Adicionalmente, a lo largo de toda la cadena intervienen diferentes agentes de gestión y mecanismos de financiación (mandatarias, obras sociales, empresas de medicina prepaga, mutuales, aseguradoras, planes asistenciales, etc.), como así también de regulación y control por parte del Estado o de entidades civiles de diverso tipo (Shigeo, 1991).

Es importante destacar que la cadena se estructura en torno a la figura de los laboratorios, quienes establecen los lineamientos en cuanto a precios, márgenes de comercialización, mecanismos de financiamiento, y retribuciones a droguerías y farmacias. En particular, los laboratorios son los principales responsables de la formación del precio del medicamento, el cual se da a conocer a través del manual farmacéutico. Si bien este precio de venta al público (PVP) es de carácter sugerido, es empleado por el resto de los actores como referencia para calcular y analizar los diferentes descuentos y márgenes de compra y venta. A su vez, los consumidores finales y los organismos de control se valen de dicha información para optimizar sus decisiones de consumo y garantizar la eficiencia en los mecanismos de mercado, respectivamente.

Según datos censados, el sector farmacéutico argentino se compone de aproximadamente 250 laboratorios nacionales y extranjeros, que abastecen fundamentalmente al mercado interno. A su vez, existen 4 grandes distribuidoras, 445 droguerías (4 principales) y alrededor de 13.000 farmacias registradas y autorizadas.

En la actualidad, las droguerías han perdido poder frente a las distribuidoras, que han asumido un rol preponderante como agentes de clasificación, control, asignación y repartición de la oferta de medicamentos. En total, la industria farmacéutica genera más de 25.000 empleos directos, a los que se le suman 100.000 empleos indirectos a lo largo de los diferentes eslabones de distribución y comercialización. Como puede observarse, la industria está integrada por una gran cantidad de agentes que interactúan entre sí. Identificarlos, estudiar su estructura de incentivos, expectativas y la naturaleza de sus relaciones resulta fundamental para comprender la complejidad del sector.

\subsection{Farmacias en Argentina - Análisis de Situación}

En el extremo final de la cadena de valor se encuentran las farmacias, cuyo número en 
Argentina asciende a alrededor de 13.000 (Tertusio, 2005, UIA, 2005).

Las farmacias se agrupan en organizaciones de diversa naturaleza y complejidad (confederaciones, colegios, cámaras, asociaciones, etc.). Hasta el año 2003, la Confederación Farmacéutica Argentina (COFA) integró y mantuvo a los distintos colegios bajo una misma representación. Posteriormente, las diferencias de intereses y estrategias dieron lugar a numerosos desprendimientos. En el año 2005, se creó la Federación Farmacéutica de la República Argentina (FEFARA), agrupando a diez colegios farmacéuticos de todo el país.

La atomización de este eslabón y el tamaño relativamente reducido de las unidades de negocio han fragmentado y debilitado su posición negociadora frente a los actores más poderosos de la oferta. Esto se evidencia, por ejemplo, en su escasa injerencia en las condiciones de compra y venta de medicamentos, establecidas principalmente por los laboratorios. Adicionalmente, las farmacias deben conceder bonificaciones a los seguros de salud (obras sociales y empresas de medicina prepaga) como parte de sus necesidades comerciales. Estos y otros factores impactan negativamente en la rentabilidad de las farmacias.

Por último, cabe destacar la importancia que han cobrado en la actualidad las modernas cadenas farmacéuticas, cuyo horario de atención se extiende por 24 horas. Ejemplos de ello son Farmacity y Vantage, y las más recientes Dr. Ahorro y Dr. Simi15 especializadas en la venta de medicamentos genéricos.

4. Asociativismo: Una aplicación a un grupo de pequeñas empresas farmacéuticas La Teoría de la Organización postula que los acuerdos de cooperación son un medio para gestionar los recursos propios de la empresa y los que son propiedad o controlados por otras, y la cooperación es la forma de acceder a ellos. Finalmente, el Enfoque Estratégico considera que las relaciones o vínculos de cooperación son una forma de poner en práctica la estrategia de la empresa (Fernandes da Silva, 2005; Martínez, 2001; Medina, Ortiz y Sacristán, 2007, citados en Ojeda, 2009).

De acuerdo con las características ya descritas del grupo de pequeñas empresas farmacéuticas en Misiones, Argentina, de analizó la aplicación del modelo asociativista con les siguientes resultados que se describen a continuación.

\subsection{Perfil Asociativista}

- Ve al competidor/proveedor como un aliado.

- Espíritu emprendedor.

- Posee gestión transparente.

- Comparte información e ideas.

- Busca resultados de largo plazo.

El Asociativismo propone a los empresarios que son desunidos y actúan individualmente, una unión capaz de hacerlos pensar colectivamente, permitiendo un intercambio de experiencias que los hará crecer en conjunto y desarrollar una cultura emprendedora.

Con el fin de hacer frente a la competencia de grandes empresas, los empresarios y sus empleados, actuando en forma conjunta tienen más oportunidades de capacitarse, y generar consecuentemente mejoras significativas en la gestión del negocio, en la calidad del trabajo y en la atención a los clientes.

"Equipo" debe ser la palabra más utilizada en toda organización, cuyo objetivo es adoptar acciones colegiadas. Jamás se debe caer en la tentación de obtener una ventaja extra e inmediata, que frustraría la filosofía 
María Luisa SaAvedra García

Fernando Paúl Molina/ Deyanira Bernal Domínguez

Cuadro $\mathrm{N}^{*}$ 2. Comparativo de figuras juridicas alternativas

\begin{tabular}{|c|c|c|c|}
\hline & Soc. Cooperativa & UTE & ACE \\
\hline TPersoneria Juridica & $\begin{array}{l}\text { Se trata de un sujeto } \\
\text { juridicamente diferente de sus } \\
\text { socios. }\end{array}$ & $\begin{array}{l}\text { No constituyen sujetos } \\
\text { juridicamente diferenciados de } \\
\text { sus participes. }\end{array}$ & $\begin{array}{l}\text { No constituyen sujetos juridicamente } \\
\text { diferenciados de sus participes. }\end{array}$ \\
\hline $\begin{array}{l}\text { 2Tipo y alcance de } \\
\text { las operaciones } \\
\text { admitidas por las } \\
\text { leyes }\end{array}$ & $\begin{array}{l}\text { Tienen duración ilimitada y son } \\
\text { fundadas para organizar y } \\
\text { prestar servicios a socios y no } \\
\text { socios. }\end{array}$ & $\begin{array}{l}\text { Las sociedades ylo } \\
\text { empresarios. mediante un } \\
\text { contrato de unión transitoria, se } \\
\text { reunen para el desarrollo y } \\
\text { ejecucion de una obra, servicio } \\
\text { o suministro concreto. Su } \\
\text { duracion será igual al de la } \\
\text { obra, servicio o suministro. }\end{array}$ & $\begin{array}{l}\text { Las sociedades yio empresarios, } \\
\text { mediante un contrato de colaboración, } \\
\text { establecenuna organización comin con } \\
\text { la finalidad de facilitar o desarrollar } \\
\text { determinadas fases de actividad } \\
\text { empresarial de sus miembros o } \\
\text { incrementar el resuitado de las mismas. } \\
\text { Tienen una duración mánima de diez } \\
\text { años, prorrogable antes de su } \\
\text { vencimiento. }\end{array}$ \\
\hline $\begin{array}{l}\text { 3Derechos y } \\
\text { obligaciones a } \\
\text { asumir por los } \\
\text { miembros entre si }\end{array}$ & $\begin{array}{l}\text { Conceden un solo voto a cada } \\
\text { asociado, cualquiera sea el } \\
\text { numero de sus cuotas sociales y } \\
\text { no otorgan ventaja ni privilegio } \\
\text { alguno a los iniciadores, } \\
\text { fundadores y consejeros, ni } \\
\text { preferencia a parte alguna del } \\
\text { capital }\end{array}$ & $\begin{array}{l}\text { Los acuerdos que deban } \\
\text { adoptar lo serán siempre por } \\
\text { unanimidad, satvo pacto en } \\
\text { contrario }\end{array}$ & $\begin{array}{l}\text { Las resoluciones, relativas a ia } \\
\text { realización del objeto de la agrupación } \\
\text { se adoptarán por el voto de la mayoria } \\
\text { de los participantes, salvo disposicion } \\
\text { contraria del contrato }\end{array}$ \\
\hline $\begin{array}{l}\text { 4La responsabilidad } \\
\text { que cada miembro } \\
\text { tendra por las } \\
\text { operaciones que el } \\
\text { grupo realice con } \\
\text { terceros }\end{array}$ & $\begin{array}{l}\text { Limitan ia responsabilidad de los } \\
\text { asociados al monto de las cuotas } \\
\text { sociales suscriptas }\end{array}$ & $\begin{array}{l}\text { Salvo disposicion en contrario } \\
\text { del contrato, no se presume la } \\
\text { solidaridad de las empresas por } \\
\text { los actos y operaciones que } \\
\text { deban desarrollar o ejecutar, ni } \\
\text { por las obligaciones contraidas } \\
\text { frente a ferceros }\end{array}$ & $\begin{array}{l}\text { Por las obligaciones que sus } \\
\text { representantes asuman en nombre de la } \\
\text { agrupación, los participantes responden } \\
\text { ilimitada y solidariamente respecto de } \\
\text { terceros. }\end{array}$ \\
\hline $\begin{array}{l}\text { 5Tratamiento } \\
\text { tributario }\end{array}$ & $\begin{array}{l}\text { Las Sociedades Cooperativas } \\
\text { son sujetos pasivos del el } \\
\text { Impuesto al Valor Agregado, } \\
\text { debiendo inscribirse como } \\
\text { Responsables Inscriptos o } \\
\text { Exentos según su cual sea } \\
\text { actividad. Tambien son sujetos } \\
\text { pasivos del Fondo para } \\
\text { Educacion y Promoción } \\
\text { Cooperativa. y }\end{array}$ & $\begin{array}{l}\text { Las Uniones Transitorias de } \\
\text { Empresas son considerados } \\
\text { sujetos pasivos del Impuesto al } \\
\text { Valor Agregado y del Impuesto } \\
\text { a los ingresos Brutos. }\end{array}$ & $\begin{array}{l}\text { Las Agrupaciones de Colaboración de } \\
\text { Empresas son considerados sujetos } \\
\text { pasivos del Impuesto al Valor Agregado } \\
\text { y del impuesto a los ingresos Brutos. }\end{array}$ \\
\hline
\end{tabular}

Fuente: Elaboración propia

del modelo. Se debe comprender que se trata de una relación en la que todos deben ganar, tanto los proveedores como los asociados.

La tentación de romper un acuerdo colectivo será menor cuando mayor sea la armonía entre los integrantes de la entidad. Más que tener compañeros, el negocio es ser compañero. Con el desarrollo de un buen compañerismo junto a los proveedores es posible obtener patrocinios para eventos, promociones y otras mejoras en los puntos de venta.

"Compartir es un fundamento básico del Asociativismo, por lo que hay que entender que la empresa es suya, pero Usted no está solo".

\subsection{Alternativas de agrupamiento}

Ante este nuevo escenario, a los pequeños y medianos empresarios farmacéuticos no les queda más alternativa que adoptar un sistema de organización que les permita comenzar a tomar decisiones en conjunto.

La pregunta que surge a esta instancia es: ¿Como comenzar a funcionar en conjunto sin perder cada uno su individualidad jurídica?

En un principio se mencionaron tres alternativas posibles: una sociedad cooperativa, una unión transitoria de empresas o bien, una agrupación de colaboración de empresas.

Las características de cada uno de ellos es posible resumirlas a continuación:

De la lectura del cuadro anterior se desprende que de las alternativas mencionadas en el presente trabajo, la figura jurídica que más se ajusta a las necesidades de un eventual grupo de empresarios farmacéuticos deseoso de comenzar a trabajar en conjunto 
pero conservando cada uno su individualidad jurídica, sería la de una Agrupación de Colaboración de Empresas.

\subsection{Estrategias Conjuntas de marketing a ser aplicadas}

4.3.1 Estrategia de marca: las farmacias del grupo pueden utilizar la misma marca única, que le permita diferenciarse de los competidores, además de permitirle lograr una importante reducción en los costos de divulgación. Es decir brinda la posibilidad de crear una marca fuerte para transmitir garantía de calidad. Disponer de una marca única posibilita también la celebración de convenios o contratos con Bancos, Empresas se Software, Agencias de Publicidad y Propaganda, Proveedores de muebles y equipamientos, etc. Otra posibilidad sería la de trabajar con una tarjeta de crédito en la que figure el nombre de la agrupación, realizar promociones de productos o servicios y comercializar productos de una marca propia.

4.3.2 Estrategia de precio: Se logra mejores precios al incrementar el volumen de compra, pudiendo al mismo tiempo comercializar a precios más bajos. En este punto corresponde aclarar que el objetivo de agruparse para incrementar el poder de negociación y de esta manera obtener mejores precios de compra y/o condiciones comerciales, no busca traducirse luego en una guerra de precios que lo único que provocaría es la de reducir significativamente la rentabilidad de todo el sector. Todo lo contrario, el objetivo es incrementar la rentabilidad de los miembros de la agrupación. Es necesario aclarar que mucho mejor que ejecutar una agresiva reducción de precios para aumentar las ven- tas, es agregar valor a los productos mediante calidad y servicio.

\subsubsection{Estrategia de canales de Marketing:} se pueden utilizar las mismas promociones e imagen para incentivar las ventas, generando grandes ahorros en este rubro. Así también,la creación de una agrupación de colaboración de empresas permite, como se dijo antes, incrementar los volúmenes de compra y por consiguiente el poder de negociación frente a los distribuidores, droguerías y laboratorios. Por lo tanto al mover un mayor volumen de compras, es posible llegar directamente a los Laboratorios como ha sucedido con las redes asociativas en el Brasil. En aquel país la resistencia inicial se transformo luego en un apoyo muy importante por parte de los Laboratorios, quienes ven con gran interés este tipo de organizaciones ya que les significa un ahorro muy importante en cuanto a gastos de comercialización se refiere. Esto es así ya que en lugar de tener que negociar con cada empresario farmacéutico en forma individual, lo hacen directamente con los representantes de la agrupación.

\subsection{Control de inventarios}

De acuerdo con la figura de asociativismo propuesta una vez obtenido una mejora en el precio y demás condiciones comerciales, cada miembro procede a comprar por separado y directamente a los Proveedores; por lo que no existen beneficios acerca del control de inventarios. Es decir, no genera beneficios a los miembros agrupados comprar por intermedio de la asociación los medicamentos ya que ello implica un encarecimiento de los mismos, pues se tendrían que incurrir en gastos administrativos y gravámenes tributarios que encarecen el producto. 


\section{CONCLUSIONES}

1. Por cuestiones tributarias proponer la aplicación de un sistema de control de inventarios con reorden y demanda incierta o bien de demanda constante por parte de un conjunto de empresas farmacéuticas en la Región del Alto Paraná Misionero, es posible pero no produce el beneficio esperado. Esto es así por el hecho de que el Código Fiscal de la Provincia de Misiones no contempla expresamente, a diferencia de la Provincia de Buenos Aires, la exención de este tipo de actividades ante el Impuesto a los Ingresos Brutos. Por lo tanto el beneficio que se obtendría inicialmente por la compra en conjunto y la aplicación de un sistema de control de inventario, se perdería luego por el pago de este último impuesto.

1. No obstante lo anterior, y a pesar de ello, subsisten las ventajas del asociativismo debido a que descartada la aplicación de un sistema de control de inventario y la compra conjunta, el mismo conserva una gran beneficio que es la posibilidad de negociar en conjunto mejores precios $y$ condiciones de pago con los proveedores. Luego, y a diferencia del caso anterior, cada miembro se encuentra posibilitado para comprar a nombre propio pero con los precios y las condiciones negociadas en conjunto.

1. Por otra parte, y en base al análisis de las normas que regulan la constitución de sociedades, la mejor alternativa de agrupamiento aplicable a un grupo de empresarios farmacéuticos dispuesto a trabajar en forma asociativa pero manteniendo su individualidad jurídica, es la de una agrupación de colaboración de empresas.

1. Este tipo de figuras jurídicas, y de acuerdo al análisis y observación de las experiencias asociativas existentes en el Brasil y en la Argentina, posibilita el desarrollo y aplicación de estrategias de marketing en forma conjunta destinadas a incrementar los márgenes y volúmenes de ventas de sus miembros a través del desarrollo de una única marca fuerte, la fijación de precios competitivos y el fortalecimiento de los canales de aprovisionamiento y distribución de los medicamentos y demás mercaderías.

\section{REFERENCIAS BIBLIOGRÁFICAS}

1. Albornoz, M. (2005). Historia [En línea]. Disponible en www.grupoharmakus.com. ar . [Fecha de consulta 23 de Septiembre del 2010].

2. Aquilano, N., Chase, R. y Jacobs, R. (2004). Administración de la producción $y$ operaciones para una ventaja competitiva, McGraw-Hill.

3. Barreira, D. (2000). Empresas-Organización Jurídica, Editorial Quórum ediciones Desalma.

4. Braidot, N. (2005). Neuromarketing Neuroeconomía y Negocios. Madrid, puerto NORTE-SUR.

5. CILFA (2006). La Industria Farmacéutica y Farmoquímica en la Argentina. Cámara Industrial de Laboratorios Farmacéuticos Argentinos (CILFA). Paris.

6. Elgue, M. (2005). Asociativismo y calidad organizacional. En las memorias del Congreso de apicultura del norte argentino, Julio de 2005, Salta.

7. Eppen, G., Gould, F., Shmidt, C., Moore, J., y Watherford, L. (2000). Investigación de Operaciones en la Ciencia Administrativa, México: Prentice-Hall.

8. Favier, E. (1988): Las uniones transitorias de empresas, Edición ERREPAR, DSE, act.12/05/1988, T.I.

9. Ibata-Arens, K., \& Obayashi, H. (2006). 
Escaping the Japanese Pyramid: The Association of Small and Medium Sized Enterprise Entrepreneurs (SME Doyukai), 1947-1999. Enterprise And Society, 7 (1), 128-163.

10. Karschenboim, R. (2001). Contratos de colaboración empresaria. Su tratamiento tributario, Edición Doctrina Tributaria ERREPAR (D.T.E.)

11. Kotler, P. y Armstrong, G. (1996): Mercadotecnia, México: Prentice Hall. Hispanoamericana, S.A.

12. Le Pera, S. (1984). Joint venture y sociedad, Edición Astrea.

13. Marsicano, A. y Marsicano, S. (2004). El Fondo para Educación y Promoción Cooperativa. A propósito de dos exenciones. Edición ERREPAR Práctica y Actualidad Tributaria (P.A.T.)

14. Moreira, G. (2005). Las pequeñas farmacias en Brasil. Brasil: Sincofarma.

15. Nissen, R. (1985). Ley de sociedades comentada, anotada y concordada, Edición Ábaco.

16. Ojeda, J. (2009). La cooperacion empresarial como estrategia de las pymes del sector ambiental. Estudios Gerenciales, 25 (110), 39-61.

17. Otaegui, J. (1984). Concentración societaria, Edición Ábaco, Buenos Aires.

18. Perciavalle, M. (2000). Manual de sociedades irregulares o de hecho, Edición Errepar.

19. Prensa Cooperativa (2006). Educación y Principios [En línea]. Disponible en www. prensacooperativa.coop/Educacion.htm. [Fecha de consulta 31 de Septiembre del 2010].

20. Romero, E. (2005). Mesa redonda rentabilidad de la farmacia, Confederación Farmacéutica Argentina (COFA), Informe del Mercado del Medicamento en Ar- gentina $4^{\circ}$ Trimestre.

21. Saavedra, M. (2011). Herramientas de planeación financiera para la Pyme. México: Gasca Sicco.

22. San Pedro, J. (1987). Manual de Organización y Gestión Cooperativa, Intercoop Editora Cooperativa, Buenos Aires.

23. Scott, H. Inicio da Rede [En línea]. Disponible en www.agafarma.com.br. [Fecha de consulta 10 de Agosto del 2006].

24. Shigeo, S. (1991). Non Stock Production: The Shingo System for continuos Improvement, Productivity Press.

25. Surraco, G. (2007). El asociativismo como estrategia pyme. Una respuesta a las necesidades de las pequeñas y medianas empresas. Question, 1 (15) 35-46.

26. Talavera, O. (2005). Como hacer una cooperativa - 6B, Edición Doctrina Tributaria Valletta Ediciones SRL.

27. Taleb, J. (2006). Bienvenidos a Uarfa [En línea]. Disponible en www.uarfa.com. [Fecha de consulta 20 de Agosto del 2006]

28. Tamascia, E. Associativismo: a solução para o varejo independente [En línea]. Disponible en www.febrafar.com.br. [Fecha de consulta 23 de Septiembre del 2005].

29. Tertusio, S. (2005). Mesa redonda rentabilidad de la farmacia, Confederación Farmacéutica Argentina (COFA), Informe del Mercado del Medicamento en Argentina. $4^{\circ}$ Trimestre

30. UIA. (2005). Cadena Farmacéutica en la Región Pampeana. Unión industrial Argentina (UIA). Mar del Plata.

31. Veron, A. (1998): Manual de sociedades comerciales, Edición Errepar, Buenos Aires.

32. Zaldívar, Manovil y Ragazzi (1997). Contratos de Colaboración empresaria, Edición Abeledo, Perrot. 